\title{
Protée
}

\section{Sophie T. Rauch}

Inquiétude de la post-humanité

\section{François Ouellet}

Volume 34, numéro 1, printemps 2006

Fortune et actualité de $\mathrm{Du}$ sens

URI : https://id.erudit.org/iderudit/013310ar

DOI : https://doi.org/10.7202/013310ar

Aller au sommaire du numéro

\section{Éditeur(s)}

Département des arts et lettres - Université du Québec à Chicoutimi

ISSN

0300-3523 (imprimé)

1708-2307 (numérique)

Découvrir la revue

Citer cet article

Ouellet, F. (2006). Sophie T. Rauch : inquiétude de la post-humanité. Protée, 34(1), 57-66. https://doi.org/10.7202/013310ar d'utilisation que vous pouvez consulter en ligne.

https://apropos.erudit.org/fr/usagers/politique-dutilisation/ 


\section{SOPHIE T. RAUCH}

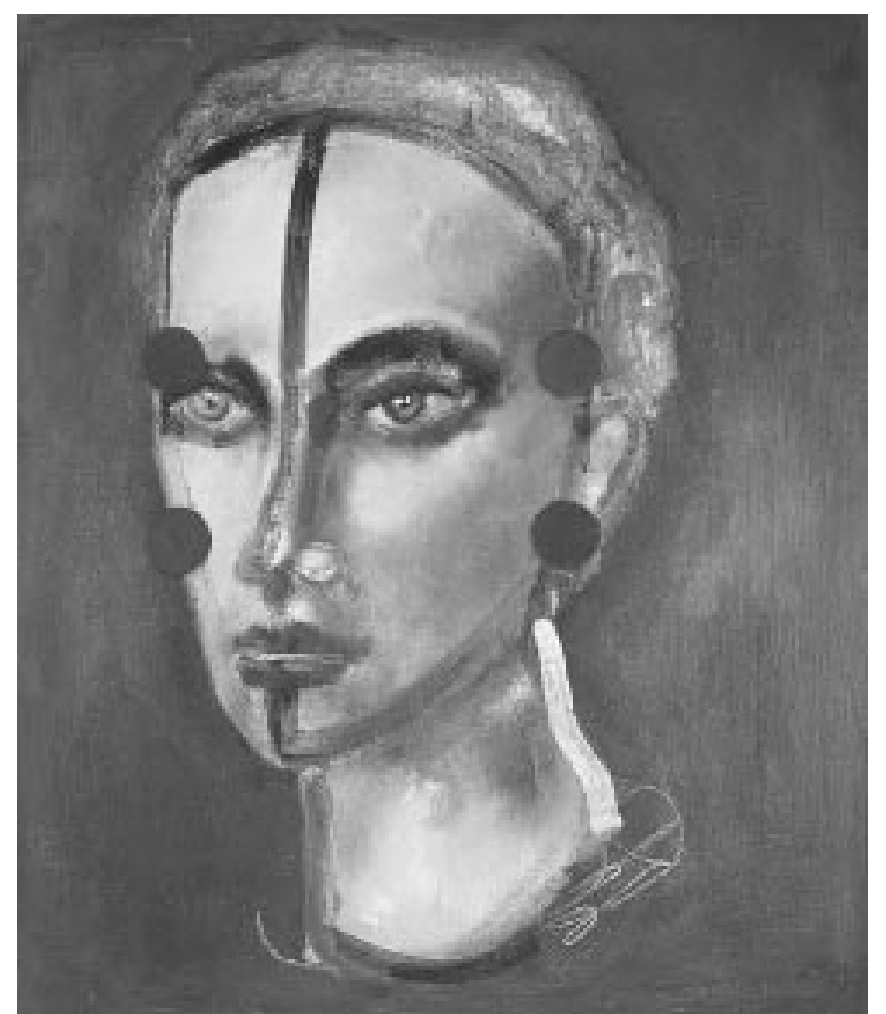

O dyssée, 2001, 49 x 42 cm. 


\section{INQUIÉTUDE DE LA POST-HUMANITÉ}

Depuis sa première exposition solo chez Madeleine Lacerte, à Québec, en 1999, Sophie T. Rauch a orienté son travail vers une appropriation et une assimilation toujours plus audacieuses du portrait, cependant qu'elle abandonnait progressivement la monumentalité du corps féminin (The Gates of Jéricho, 1997), les nombreuses références à l'histoire de l'art ou encore ces évocations de têtes et de corps qui rappellent irrésistiblement la manière et les couleurs d'Odilon Redon (Welcome to Paradise, 1998), les toiles largement abstraites au sein desquelles ressortaient un œil clairement dessiné (Survol, 1995) ou l'esquisse d'un visage (Das Wissen, 1995). Il y avait dans cette peinture tout un travail sur l'histoire et la mémoire de l'art, où une forme de spiritualité était traversée par un imaginaire de la dissolution, de la décomposition des corps et des repères spatiaux.

Il importe d'évoquer ces œuvres, car elles nous permettent, par contraste, de mieux saisir l'évolution du travail de Sophie T. Rauch. Dans la production qu'elle exposait chez Han Art, à Montréal, en 2002, le portrait est largement dominant. Ce sont presque toujours des visages de femmes au regard fixe; souvent, un œil est traité $d^{\prime}$ 'une manière singulière, comme c'est le cas dans Almost There (2001), où le regard et la bouche sont brouillés, peutêtre en raison d'une source lumineuse oblique, et dans $\mathrm{Half}$ and $\mathrm{H}$ alf (2001), titre qui explicite le travail géométrique de I'artiste. II semble que chez Rauch I'œil importe davantage que les yeux, comme le signifiait déjà cette obsession de l'œil détaché du visage dans la production antérieure (Watching I - Watching Eye, 1996). Une toile comme Doll (2001) reproduite ici en page couverture -, qui reprend ni plus ni moins $A 1598 R$, une œuvre de 1998, mais en lui faisant subir une distorsion particulière, déconstruit littéralement le regard en multipliant l'œil sur une ligne horizontale - mais aussi la bouche et le nez subissent le même traitement-, comme si nous avions accès au brouillon de la toile, au travail de l'artiste, lequel serait fait de sélections et de calculs, comme veulent peut-être le suggérer les lettres et les chiffres qui encadrent le visage de la femme. Au-delà de cette lecture esthétique imposée par la toile, I'isolement des parties du visage insiste sur les sens (la vue, le goût et l'odorat). On observe aussi que le personnage de Doll, à l'exemple de la production antérieure, est bordé d'un fin tracé perlé; à cet égard, cette toile fait exception dans la production 2001, où l'artiste continue de délimiter les visages mais par la 
présence de cercles massifs (par exemple Odyssée, 2001), comme si la tête perdait de son autonomie au profit d'un chiffrage du sujet. Dans une toile comme I don't really know about this (2001), les cercles sont situés au-dessus de la tête, ce qui suffit à déplacer la valeur humaine vers I'abstraction de la reproduction formelle: la tête devient socle, récipient - caractéristique propre à l'ensemble des portraits de cette période. Dans tous les cas, l'évolution du travail de l'artiste approfondit la vague inquiétude qui caractérisait les premières toiles; le visage semble s'être robotisé, définitivement déshumanisé (The Final Frontier, 2001, The Cage, 2002). Dans Robotic Ingres (2002), les tresses semblent être des ressorts bien fermes, comme si la tête, sorte de métonymie du sujet en perte d'humanité, n'était plus mue que par une troublante mécanique élastique. Nous sommes ici confrontés à cette espèce d'individu «clonal» évoqué par Baudrillard, un individu pris dans les mailles du réseautage informatique, sans relief politique ou subversif et dépourvu de toute altérité.

Ce travail du portrait, Sophie T. Rauch le poursuit patiemment dans sa production la plus récente, celle exposée à la Galerie Orange, à Montréal, en 2005. Ici les visages sont constitués par des

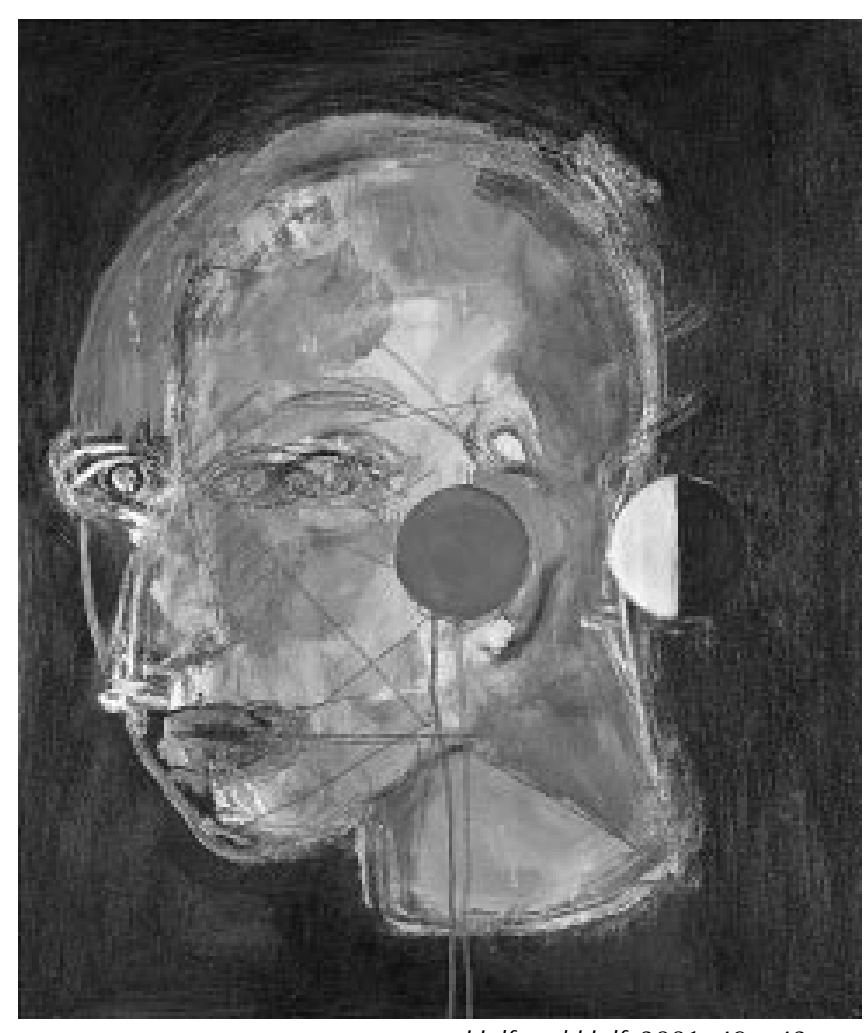

Half and Half, 2001, $49 \times 42 \mathrm{~cm}$.

taches multicolores à la manière pointilliste. Le contour des yeux, l'arête du nez, les lèvres tendent à se perdre dans l'ensemble (Vortex, 2005), la tête, elle-même délimitée par les taches de couleur, n'étant plus qu'une vaste constellation (Cassiopée, 2005), une figure de l'éternité minéralisée et insécable. La perte de sens s'est approfondie, l'inachevé et l'éphémère dominent; les titres des toiles sont révélateurs d'une autre dimension à retrouver: Searching for Tomorrow, Victoria Génétique, À la recherche du temps perdu, Vertigo - toutes des œuvres de 2004 -, Metropolis (2005). Au-delà de
I'inquiétude, quelque chose résiste donc, qui relève peut-être de l'énigme et de l'interprétation - comme aux temps mythologiques -, qui témoigne d'une sorte de clivage épistémologique, d'une sémiotique du désir, d'une signification à réapprivoiser et à habiter.

François O uellet 


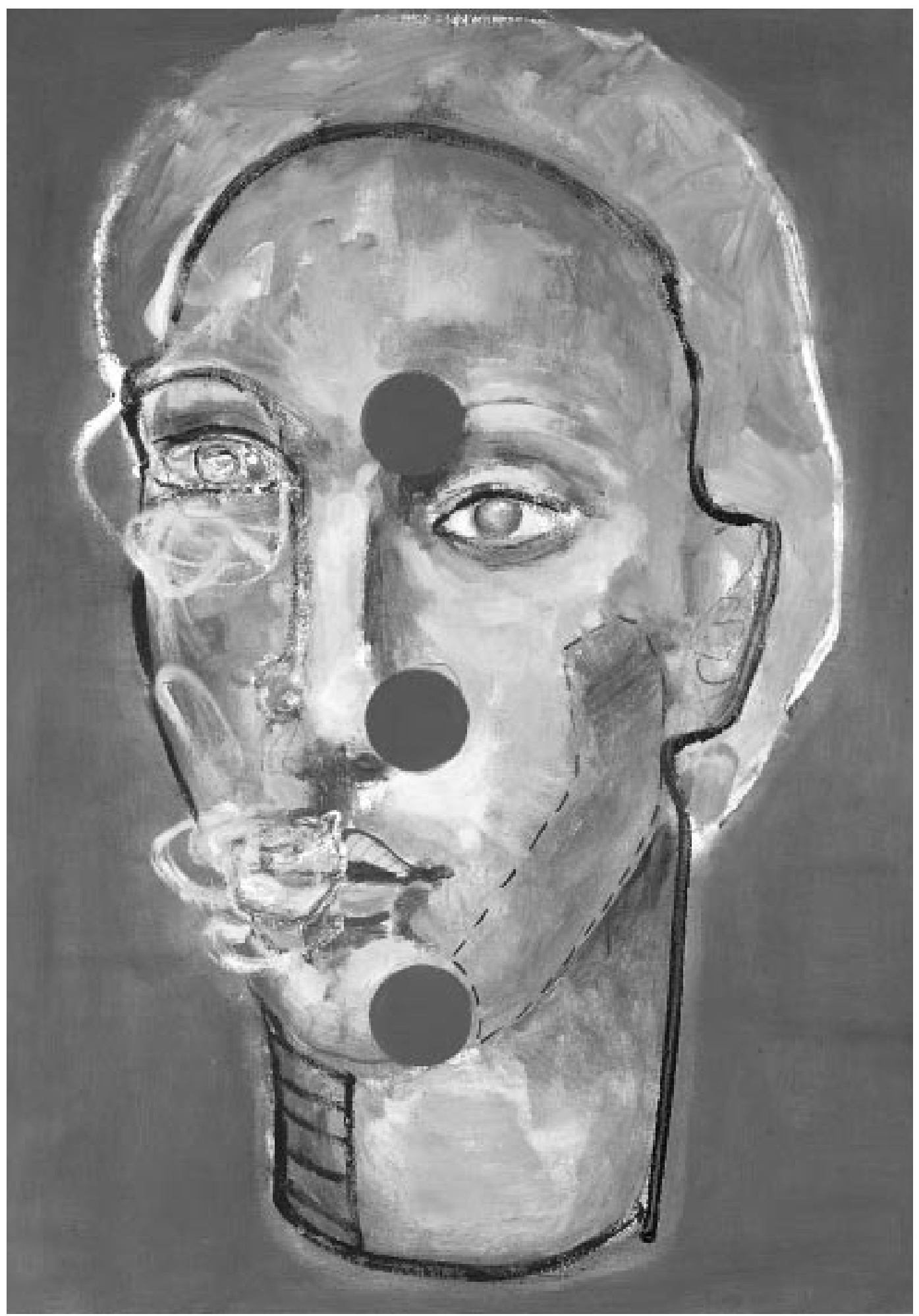

Almost There, 2001, $87 \times 51 \mathrm{~cm}$. 


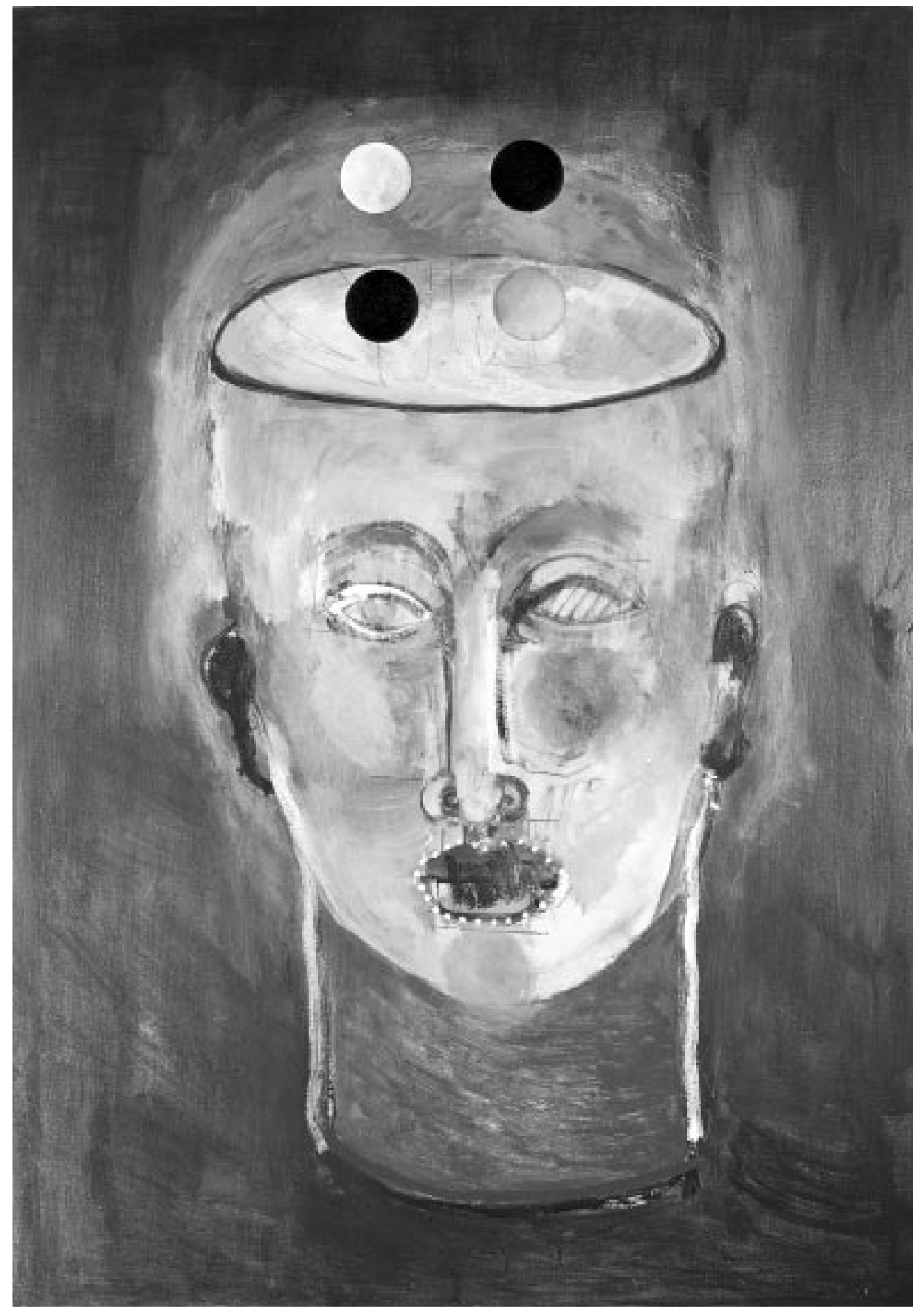

I dont't really know about this, 2001, $115 \times 80 \mathrm{~cm}$. 


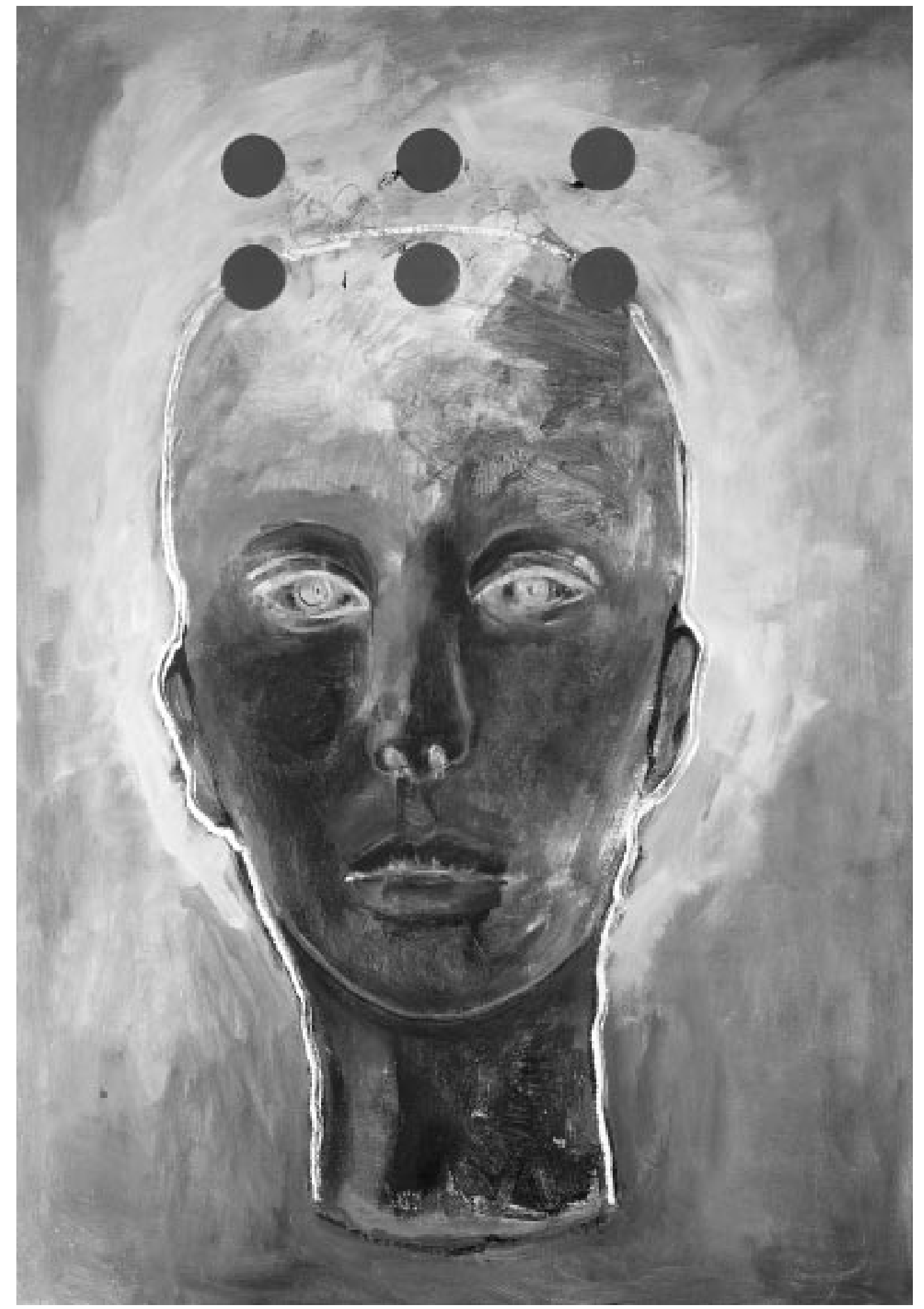

The Final Frontier, 2001, 115 x $80 \mathrm{~cm}$. 


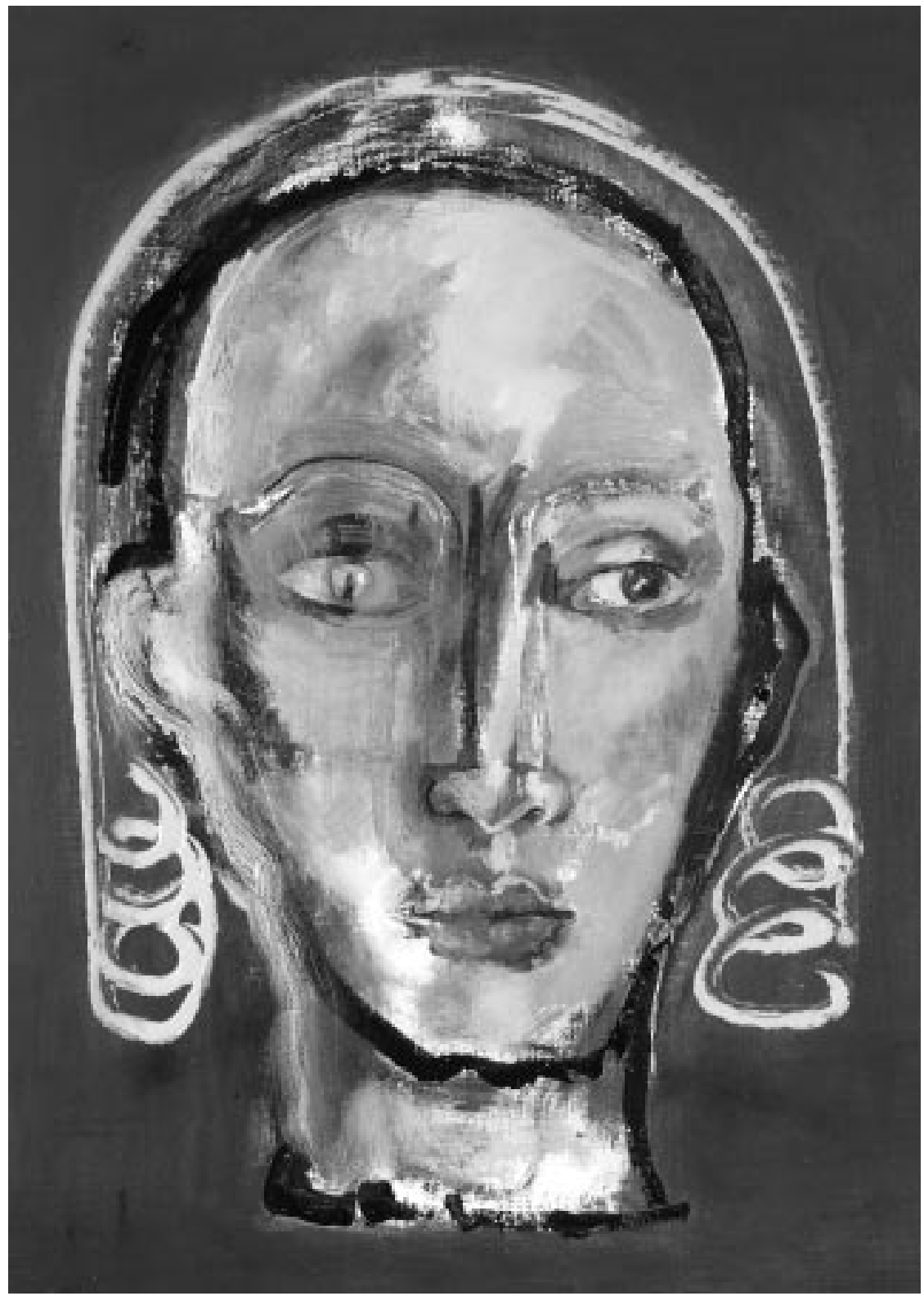

Robotic Ingres, 2002, $49 \times 42 \mathrm{~cm}$ 


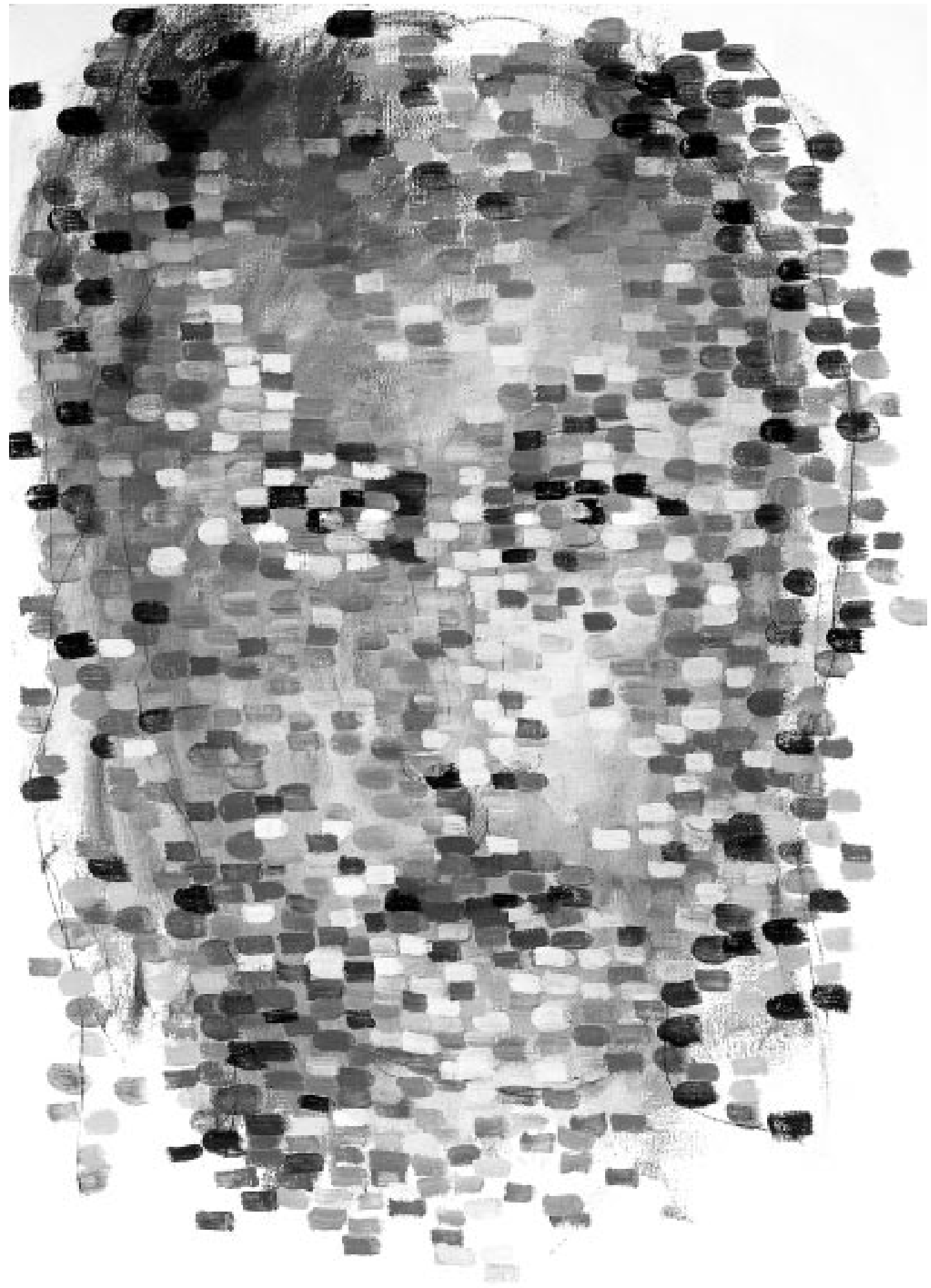

Vortex, 2004, $80 \times 57 \mathrm{~cm}$. 


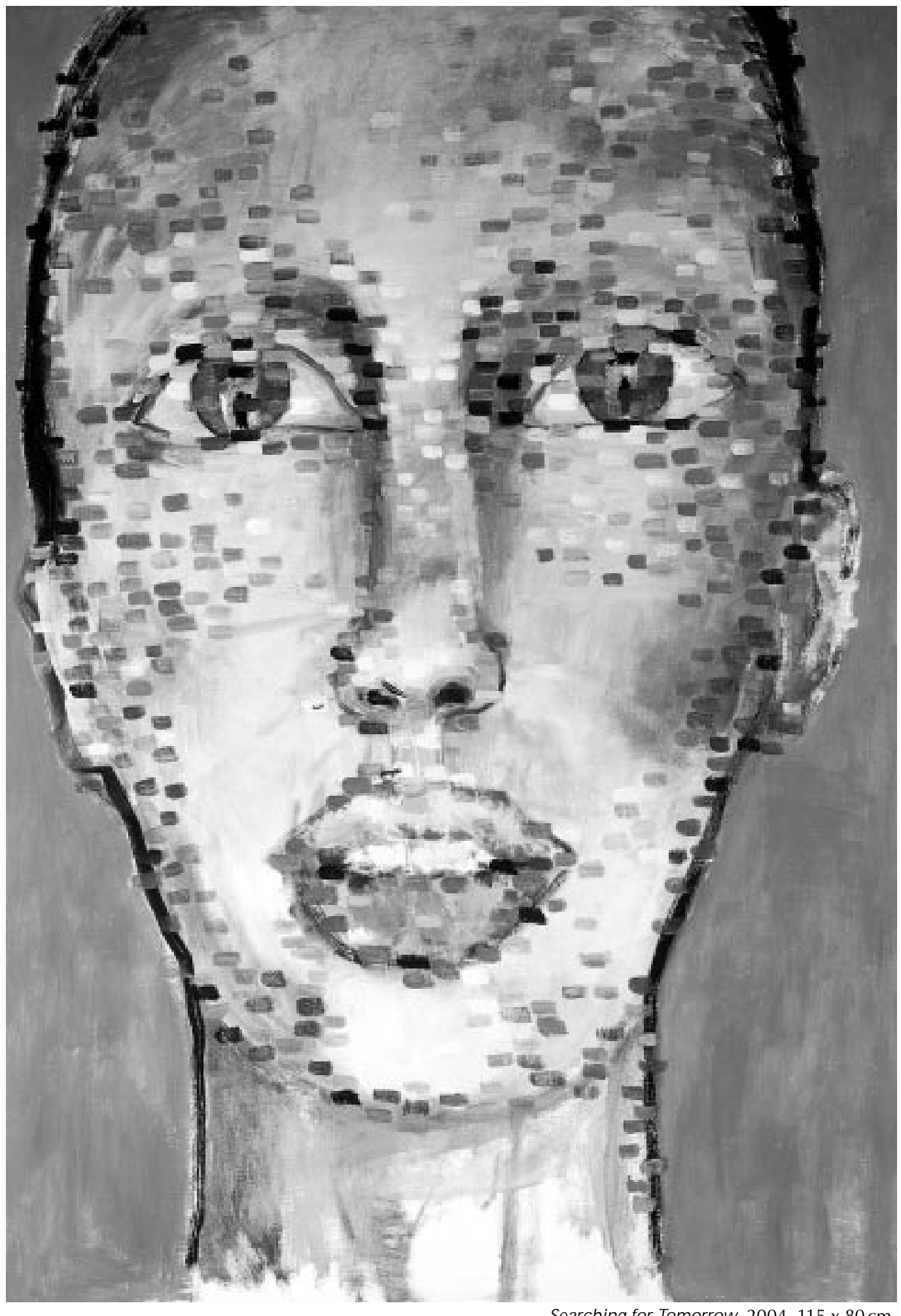

Searching for Tomorrow, 2004, 115 x $80 \mathrm{~cm}$. 


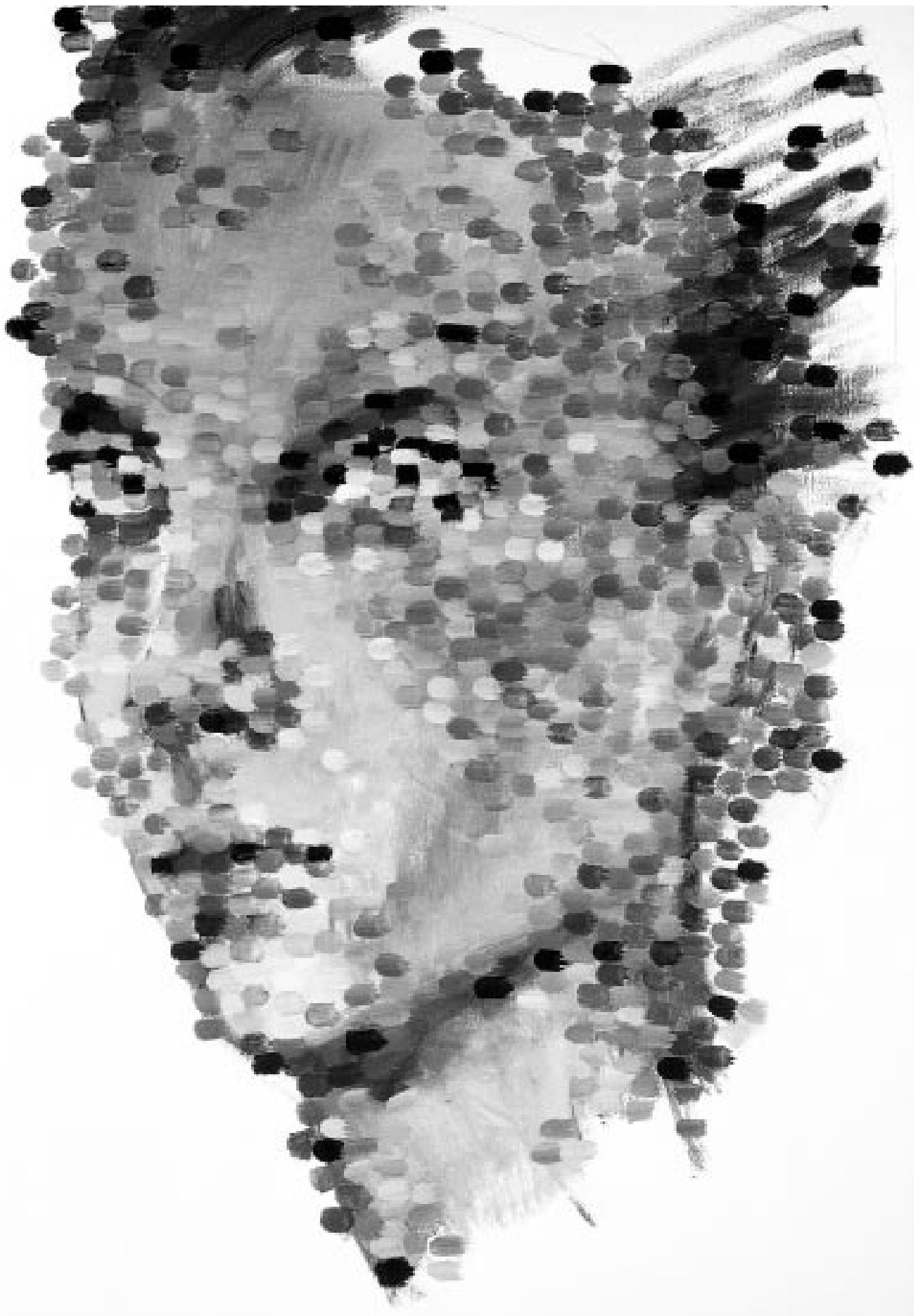

Quantum Leap, 2005, 115 x $80 \mathrm{~cm}$. 